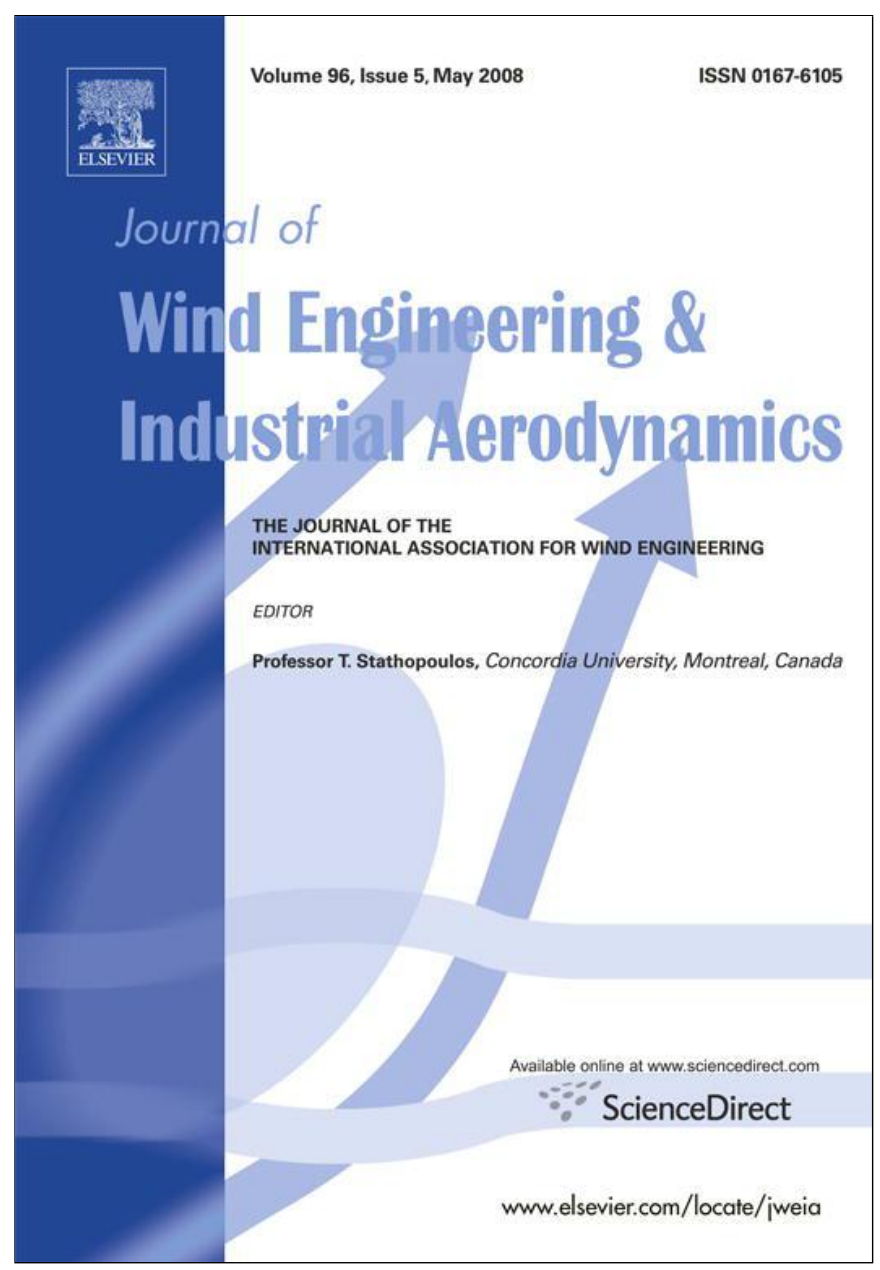

This article appeared in a journal published by Elsevier. The attached copy is furnished to the author for internal non-commercial research and education use, including for instruction at the authors institution and sharing with colleagues.

Other uses, including reproduction and distribution, or selling or licensing copies, or posting to personal, institutional or third party websites are prohibited.

In most cases authors are permitted to post their version of the article (e.g. in Word or Tex form) to their personal website or institutional repository. Authors requiring further information regarding Elsevier's archiving and manuscript policies are encouraged to visit:

http://www.elsevier.com/copyright 


\title{
The numerical computation of near-wall turbulent flow over a steep hill
}

\author{
Juliana B.R. Loureiro ${ }^{\mathrm{a}, \mathrm{b}, *}$, Alexandre T.P. Alho ${ }^{\mathrm{c}}$, \\ Atila P. Silva Freire ${ }^{\mathrm{a}}$ \\ ${ }^{a}$ Mechanical Engineering Program (COPPE/UFRJ), C.P. 68503, 21945-970 Rio de Janeiro, Brazil \\ ${ }^{\mathrm{b}}$ Scientific Division, National Institute of Metrology (INMETRO), 22050-050 Rio de Janeiro, Brazil \\ ${ }^{\mathrm{c}}$ Naval and Ocean Engineering Department (Poli/UFRJ), 21945-970 Rio de Janeiro, Brazil
}

Received 4 January 2006; received in revised form 19 April 2007; accepted 18 January 2008

Available online 18 April 2008

\begin{abstract}
The present work performs a detailed comparison between numerical computations for the flow over a two-dimensional steep hill and some newly obtained laboratory data. Six turbulence models were tested:

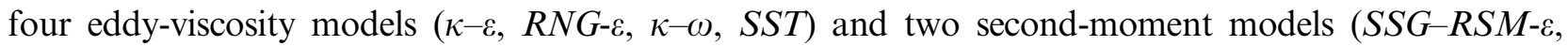
$B S L-R S M-\omega)$. The experiments were conducted in a water channel and were specially planned such that the large separated flow region that is formed on the lee side of the hill could be well scrutinized. The experimental results include complete profiles of the mean velocity components and of the twodimensional Reynolds stress tensor and were obtained through the laser Doppler anemometry. A particular concern of this work has been to achieve a detailed experimental and numerical characterization of the near-wall flow region. As such, for most of the measuring stations, at least eight points were located in the viscous sublayer. The work also shows the distribution of wall-shear stress in detail. The $\omega$-equation-based models were observed to perform much better than the $\varepsilon$-equationbased models. The length of separated flow region, mean velocity profiles and wall-shear stress were all reasonably well predicted. The flow properties on the hill top were particularly difficult to describe. The turbulence properties in the reversed flow region were best simulated by the $B S L-R S M$ model.
\end{abstract}

(C) 2008 Elsevier Ltd. All rights reserved.

Keywords: Numerical computations; Flow over hills; Experiments; Skin friction

\footnotetext{
${ }^{*}$ Corresponding author at: Mechanical Engineering Program (COPPE/UFRJ), C.P. 68503, 21945-970 Rio de Janeiro, Brazil. Tel./fax: + 552125627748 .

E-mail addresses: jbrloureiro@gmail.com (J.B.R. Loureiro), alho@peno.coppe.ufrj.br (A.T.P. Alho), atila@mecanica.coppe.ufrj.br (A.P. Silva Freire).
} 


\section{Introduction}

The number of works devoted to the numerical computation of flows over surfaces steep enough to provoke flow separation has been significant over the last few years. The reason for this is quite clear. In addition to the variety of practical applications this problem has, the large increase in the computational capabilities of softwares dedicated to handle specific subjects has meant that problems that were previously considered a great challenge can now be solved with some comfort. In particular, solution procedures are now based on turbulence models that have been developed to a great degree of sophistication and on numerical procedures that attain a fair degree of generality. Still, in many cases, the numerical data that is produced require some rigorous and detailed validation.

For the turbulent flow over a steep hill, the experimental validation of numerical predictions is of utmost importance. Indeed, the large separated flow region that is formed on the lee side of the hill results in some flow characteristics that are difficult to simulate numerically and that, therefore, require rigorous validation. The correct prediction of the flow separation point, of the extent of the separation bubble and of the turbulence production are typical examples of difficult problems. Even on the experimental front the difficulties are many. The elevation of the wall together with the induced reverse flow pose problems that are difficult to overcome. For example, the hot-wire anemometer will not be an adequate choice for the characterization of the velocity field since it will not be able to discriminate the flow direction. Preston tubes or pulsed-wires will not fit to the wall curvature, making it a difficult affair to find the wall-shear stress. The result is that, for flows over a steep hill, specific numerical and experimental techniques need to be devised.

A particularly difficult problem to treat is the specification of boundary conditions at the wall. Irrespective of the type of turbulence closure scheme that is chosen, the large gradients in flow variables in the near-wall region claim for a special near-wall treatment, preferably simple and not expensive in terms of computational effort. The possible alternatives are many, including the specification of wall functions and of low-Reynolds number corrections. Thus, flow numerical simulations will clearly depend on implementation decisions. Therefore, a critical choice, and probably the main source of error in simulations, is the specification of the near-wall model.

The purpose of the present work is to make a judicious study on the use of turbulence models for the description of a separated flow around a hill. In special, we will be concerned with the near-wall flow description. Here, we should remind the reader that near-wall data sets for the separated flow region on the lee of a hill are not easy to find. The recent works of Kim et al. (1997), of Ishihara et al. (2001) and of Ross et al. (2004), for example, present comprehensive experimental studies on the flows over steep hills. However, the near-wall region is not well characterized in none of these works. Therefore, a second objective of this work is to obtain near-wall data that are appropriate enough to a good model validation.

To find specific data for the near-wall properties of the flow over a steep hill, a single experiment was carried out in the Hydraulics Laboratory of the Civil Engineering Department of Oporto University. Using laser Doppler anemometry, the flow over a steep hill was characterized at 13 positions in a water channel. In particular, seven measuring stations were used to characterize the flow separated region. The experiments were sufficiently detailed to provide data of mean and fluctuating velocity components in the near-wall viscous region; at least eight points have been located in the first $3 \mathrm{~mm}$ away 
from the wall. Moreover, the data, upon further processing, provided results for the mixing-length and eddy-viscosity distributions, wall-shear stress and turbulence production. The overall implication is that these data form a very comprehensive set to which numerical computations of near-wall flow over a hill can be tested.

Thus, the present very detailed experimental characterization of the near-wall flow properties will permit an assessment of turbulence models against some flow parameters that are of difficult evaluation, e.g., the wall-shear stress. The tested models will include eddy-viscosity and full second-moment models, namely: four eddy-viscosity models, (i) the standard $\kappa-\varepsilon$ model, (ii) the $\kappa-\omega$ model, (iii) the shear stress transport model (SST- $\omega$ ), (iv) the renormalization group model $(R N G-\varepsilon)$; and, two Reynolds stress models, (v) the $S S G-R S M$ model, (vi) the baseline- $\omega$ Reynolds stress model (BSL- $\omega$ ).

Flows over curved surfaces offer a difficult test to turbulence models. Indeed, Wang et al. (2004) have pointed out how difficult it is to correctly predict flow separation from curved surfaces. Furthermore, these authors have commented that much of the effort concerning the investigation of turbulence closures does not tackle separation provoked by smoothly varying adverse pressure gradients. Computations over a two-dimensional hill show that most of the considered models over-estimate the size of the recirculation region. This fact is argued to be associated with an insufficient level of shear stress in the separated shear layer. For computations over a three-dimensional hill, none of the models are observed to perform well.

The difficulties posed by near-wall models have been vastly reported in literature (see, e.g., Ying and Canuto, 1997; Iizuka and Kondo, 2004). In practical problems, a common approach is to resort to approximate expressions for each near-wall variable. This procedure avoids a detailed numerical treatment and the uncertainties introduced by the specification of a turbulence model. In second-order models, modifications are sometimes considered in the pressure-strain term and in the diffusion term. However, in many other circumstances, wall functions and modified modelled turbulence equations do not suffice to resolve the resulting difficulties. As an alternative approach, low-Reynolds number models have been developed by some authors by incorporating in the formulation a wall damping effect. Several low-Reynolds number models can be found in literature, including the $\kappa-\omega$ model. All these changes may lead to model sensitivity to free stream conditions. Thus, taking into respect the strengths and the weaknesses of the several proposed models, an optimization of their performance can be achieved by an explicit consideration of their best zonal characteristics. This solution has been adopted by many of the new proposed models with the introduction of blending functions. The present work offers a strong test to these models in view of the newly available near-wall data.

The application of numerical models for the description of turbulent flows over hills has been particularly significant over the last 10 years. The flow over a two-dimensional hill covered by a forest canopy was studied numerically by Kobayashi et al. (1994). The averaged Navier-Stokes equations were closed with the extended $\kappa-\varepsilon$ model of Svensson and Haggkvist (1990) that considers two extra terms to account for the drag caused by the canopy. To eliminate false diffusive errors, the authors resorted to a finite-volume numerical algorithm with a highly accurate numerical scheme constructed from a class of total variation diminishing (TVD) schemes. The predicted numerical results were compared with the reported LDV wind tunnel measurements of Ruck and Adams (1991). Velocity profiles showed a reasonable good agreement except for the measuring station right at the half-way on the lee side. Comparison of turbulent kinetic energy and of 
turbulent shear stress profiles, however, was very poor. Typical grid size was $100 \times 60$. The wall boundary conditions were applied through the classical law of the wall.

Hurley (1997) conducted a numerical assessment of 10 different local turbulence closure procedures ranging from first-order to two-equation prognostic schemes. His simulations included two-dimensional sea-breeze flow and flow over a hill. The author concluded that if just mean meteorological fields are to be predicted, then, any of the considered models will produce reasonable results. If, on the other hand, turbulent fields are required, twoequation models should be used.

A computation of turbulent flows over two-dimensional hills with different slopes was carried out by Ying and Canuto (1997) through a second-order closure model. The simulations used a finite-difference method and a non-hydrostatic atmospheric model. The results were compared with the EPA wind tunnel data from the RUSHIL experiment (Khurshudyan et al., 1981) and with lower level turbulence models, including an eddyviscosity model and an algebraic Reynolds stress model. Inasmuch previous authors, Ying and Canuto concluded that mean velocity profiles are insensitive to the closure model that was used. However, second-order closure models are reported to perform better in capturing variations in turbulent intensities.

The RUSHIL experiment was also simulated numerically by Castro and Apsley (1997). Turbulence closure was achieved through a suitably modified $\kappa-\varepsilon$. Again, the model was shown to produce good agreement for the mean fields, but lower values for the turbulent kinetic energy and the lateral plume spread. The corrections in the standard $\kappa-\varepsilon$ model allowed the authors to account for streamline curvature effects. For the hill with a large separated region, the levels of concentration were found to be well predicted. For hills with lower slopes, with intermittent separation, less satisfactory results were observed.

The flow over Blashaval hill was numerically simulated by Hewer (1998) using a nonlinear model. The main purpose of the work was to assess the model behaviour on the leeslope. The Reynolds stresses were modelled through two eddy-viscosity models that used the mixing-length and the turbulent kinetic energy as reference scales. On the upwind slope, results for the non-linear and linear models were comparable. On the lee-slope, however, the non-linear model overpredicted the wind speeds but performed much better than the linear model. The difficulties in dealing with the lee side of the hill arose from inaccuracies in the simulation of flow separation.

The usefulness of two-equation turbulence models that appeal to isotropic eddyviscosity and wall functions was studied by Kim and Patel (2000). Five turbulence models were tested for the neutral boundary layer on a flat surface and two- and three-dimensional models and real terrain. The best predictions with respect to flow profiles and length of separated flow region were achieved by the RNG-based $\kappa-\varepsilon$ model.

Castro et al. (2002) investigated the importance of spatial discretization and the limitations of the $\kappa-\varepsilon$ turbulence model in the numerical simulation of neutrally stratified flow over Askervein Hill. To capture the relevant flow features on the lee side of the hill, a time-dependent formulation and a third-order discretization of the advection terms were required. At the hill top, the predicted speed-up factor was $10 \%$ smaller than the experimental value. The characteristic roughness near the hill top was reduced so as to improve the agreement between numerical and experimental data.

The performance of large-eddy simulations to the description of turbulent flow over a two-dimensional steep hill was evaluated by Iizuka and Kondo (2004). Four different subgrid scale (SGS) models were tested together with two different ground surface 
conditions. The computations were compared with the experiment of Ishihara et al. (2001). The dynamic SGS models furnished results that were observed to be in very poor agreement with the experimental data; this was, according to authors, due to the inaccurate estimation of the near ground surface model coefficient. To improve accuracy, a hybrid SGS model was advanced by the authors.

Two-dimensional steep hills in both neutral and stably stratified flow conditions were also studied by Ross et al. (2004). Turbulence models that used one-and-a-half and secondorder closure schemes were used to predict the mean and turbulent quantities of the flow. The numerical predictions were compared to new wind tunnel experiments carried out for two hills with different slopes, one of which was steep enough to cause flow separation. The data, obtained through laser Doppler anemometry, included mean and turbulent properties of the flow. The numerical simulations were conducted in a two-dimensional domain with $128 \times 80$ grid points. The wall flow region was treated accordingly to the procedure of Ying and Canuto (1997). The authors report a reasonable prediction for mean flow characteristics for all flow conditions. However, large differences are observed in the separated flow region in the lee side of the hill.

The ability of non-linear eddy-viscosity and second-moment models to describe the flow over two- and three-dimensional hills was investigated by Wang et al. (2004). Five turbulence models were analyzed: two cubic eddy-viscosity models, an explicit algebraic Reynolds-stress model, a quadratic eddy-viscosity model and a Reynolds-stress-transport model. The one major objective of the paper was to examine the flow separation patterns that occur on the lee side of two-dimensional- and three-dimensional-hills. The authors report that in two-dimensional-flow the predicted separation differs greatly from one model to the other, with just one non-linear model performing well. In three-dimensionalflow, none of the models were found to give a good representation of the complex multivortical separation pattern. For the two-dimensional- and three-dimensional-flows typical grid sizes were $700 \times 90$ and $110 \times 105 \times 80$ nodes, respectively.

\section{Experimental set-up and flow conditions}

\subsection{Description of water-channel and of laser-Doppler system}

The measurements were carried out in an open-channel in the Hydraulics Laboratory of the Civil Engineering Department, University of Oporto, Portugal. The water channel has a test section $40 \mathrm{~cm}$ wide, $60 \mathrm{~cm}$ high and $17 \mathrm{~m}$ long. The recirculation system consists of two return tanks, an elevated stabilization tank, and four pumps with maximum total capacity of $150 \mathrm{l} / \mathrm{s}$. The working section was fitted with glass side walls, $3 \mathrm{~m}$ in length, and was situated $7.3 \mathrm{~m}$ downstream of the channel entrance. The model hill top was located at $8 \mathrm{~m}$ away from the channel entrance.

In a typical run the system could be operated with a maximum flow rate variation of $\pm 0.8 \%$. At the entrance of the channel, a series of screens and filters were used to suppress any excessive level of turbulence. A magnetic flowmeter installed in the supply line was used to control the flow rate. The water depth along the channel was controlled by a vertical steel gate fitted at the outlet section. The traversing system was three-dimensional, very sturdy, independent of the water channel, and had a positioning precision of $\pm 10 \mu \mathrm{m}$.

The velocity components, mean and fluctuating, were obtained through a one component, fiber optic, Dantec laser-Doppler anemometry system that was used in the 
forward scatter mode. The laser source was a $2 \mathrm{~W}$ Ar-ion operating in multi-mode. To resolve the direction of the flow and to correctly measure near-zero mean velocities a Bragg cell unit was used with an electronic shift of $0.6 \mathrm{MHz}$. The measurement volume was positioned on the channel centreline with the help of lenses with a focal length of $310 \mathrm{~mm}$ that were mounted on the probe. Before hitting the photomultiplier, the scattered light passed through an interference filter of $514.5 \mathrm{~nm}$, so that only green light was acquired. The signal from the photomultiplier was band-pass filtered and processed by a TSI 1990C Counter, operating in single measurement per burst mode. A series of LDA biases were avoided by adjusting the strictest parameters on the data processor. For each point measured, a sample size of 10,000 values has been considered. Table 1 lists the main characteristics of the laser-Doppler system used.

This system was used to measure both the longitudinal and the vertical velocity components. This was easily made by simply turning the probe around its axis, so that, on both conditions, the fringe distribution was perpendicular to the measured velocity component. As for the Reynolds shear stresses, measurements were made by turning the probe to the positions $\pm 45^{\circ}$ according to the procedure described by Logan (1972). Typical uncertainties for the mean velocity components $U$ and $W$ in the undisturbed flow region are lower than $0.2 \%$ of the free stream velocity, $U_{\delta}$. Downstream of the hilltop, in high level turbulence regions, these uncertainties increase to about $0.3 \%$ of the free stream velocity. For the fluctuating quantities, $(\overline{u u})^{1 / 2},(\overline{w w})^{1 / 2}$, and $-\overline{u w}$, the estimated uncertainties in the undisturbed flow region are of $2.3 \%, 1.8 \%, 4.2 \%$ of the friction velocity in the undisturbed flow (for the Reynolds shear stress the uncertainty is given in percentage of the square of the friction velocity of the undisturbed flow), respectively, increasing to $3.8 \%, 3.5 \%$ and $6.9 \%$ in regions of high turbulence.

\subsection{Details of elevation}

The wall elevation studied in the present work was two-dimensional and aerodynamically smooth. In fact, the shape of the elevation described below is much similar to those studied by other authors (see e.g., Britter et al., 1981; Arya et al., 1987). The chosen elevation followed a modified "Witch of Agnesi" profile, according to equation

$$
z_{H}(x)=H_{1}\left[1+\left(x / L_{H}\right)^{2}\right]^{-1}-H_{2} .
$$

Hence, $H\left(=H_{1}-H_{2}\right)(=60 \mathrm{~mm})$ is the hill height and $L_{H}(=150 \mathrm{~mm})$ is the characteristic length of the hill representing the distance from the crest to the half-height point. The rectangular Cartesian co-ordinates are given by $x$ and $z$.

Table 1

Main characteristics of the laser-Doppler system

Wavelength

Half-angle between beams

Fringe spacing

Frequency shift
$514.5 \mathrm{~nm}$

$3.415^{\circ}$

$4.3183 \mu \mathrm{m}$

$0.60 \mathrm{MHz}$

Dimensions of the measurement volume 
The present hill is steep enough to promote a large flow recirculation region on the lee side of the elevation. The model was constructed from a sheet of polished plexiglass. The maximum slope, $\theta_{\max }$, is $18.6^{\circ}$.

\subsection{Measuring stations}

Measurements were made on the channel centreline at the 13 stations shown in Fig. 1. The origin of the rectangular Cartesian coordinate system should be noted. The data presentation will take the hill height and the undisturbed external boundary layer mean velocity as reference parameters. The properties of the undisturbed profile are shown in Table 2.

\subsection{Wall-shear stress}

For attached flows, chart methods based on the log-law can be used to find the wallshear stress. Alternatively, the identification of an existing constant shear stress wall layer can also be used to determine the wall-shear stress. As soon as a reversed flow region sets in, the validity of these procedures breaks down making the estimation of wall-shear stress a much difficult affair. In fact, in most of the available publications, graphs of wall-shear stress include only attached flow regions and are presented with very brief explanations on

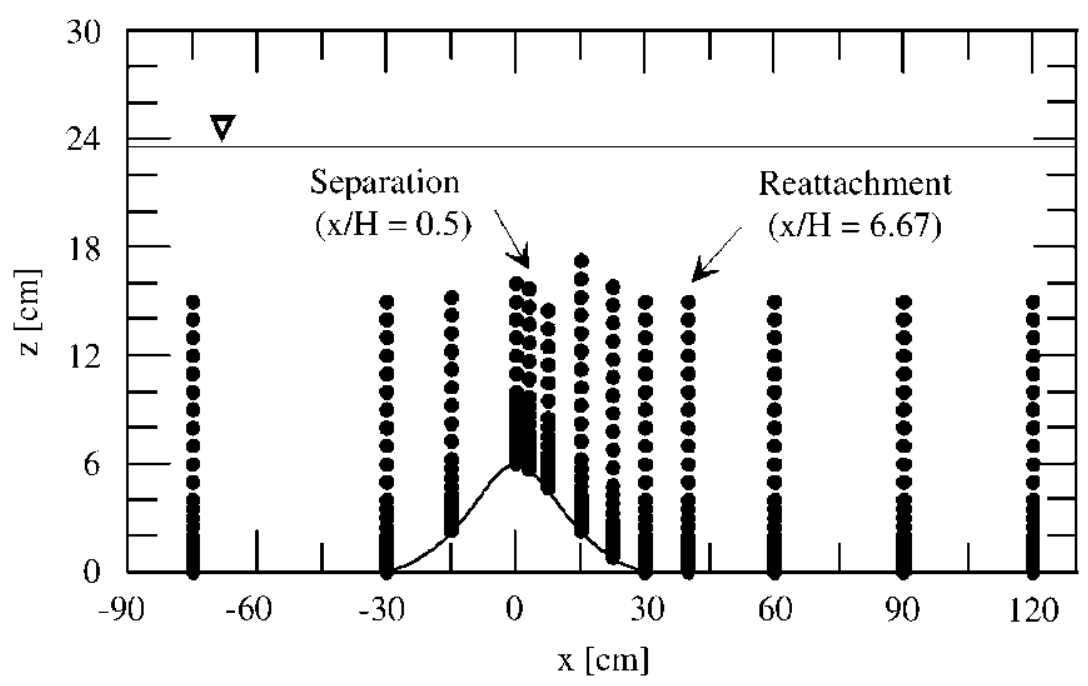

Fig. 1. Position of measuring stations and co-ordinate system.

Table 2

Properties of undisturbed profile

\begin{tabular}{lll}
\hline Boundary layer thickness & $\delta$ & $100 \mathrm{~mm}$ \\
External velocity & $U_{\delta}$ & $0.0482 \mathrm{~m} / \mathrm{s}$ \\
Reynolds number & $R_{\delta}$ & 4772 \\
Friction velocity & $u_{*}$ & $0.0028 \mathrm{~m} / \mathrm{s}$ \\
Roughness length & $z_{0}$ & $0.27 \mathrm{~mm}$ \\
Longitudinal velocity fluctuations $(z / \delta=0.05)$ & $(\overline{u u}) / u_{*}^{2}$ & 2.50 \\
Transversal velocity fluctuations $(z / \delta=0.08)$ & $(\overline{w w}) / u_{*}^{2}$ & 0.83 \\
\hline
\end{tabular}


how they were constructed. Here, a more detailed explanation will be provided. For a complete account of the wall-shear stress evaluation for the flow over the model hill considered in this work the reader is referred to Loureiro et al. (2007a).

The Clauser chart method estimates wall-shear stress by relying on the hypothesis that in the boundary layer the mean velocity profile assumes a logarithmic form at a certain distance from the wall. Then, by plotting the data in a log-form and by considering von Karman's constant to be known, wall-shear stress can be evaluated directly from the slope of the resulting straight line. In a separated flow region, the near-wall velocity will not follow a logarithmic profile. That means some alternative technique has to be used to find the wall-shear stress.

Consider that in a turbulent boundary layer the very near-wall region is dominated by viscous effects. Then, under adverse pressure gradient conditions, the momentum equation in the viscous sublayer is dominated solely by the viscous and pressure terms. A double integration of this equation furnishes a second degree polynomial relationship between the velocity and the distance from the wall as shown by Eq. (2). Here, the notation is classical; $\tau_{*}=\rho u_{*}^{2}$ denotes the wall-shear stress. Thus, provided detailed measurements are made in the viscous region, Eq. (2) can be used to find not only the wall-shear stress but also the local longitudinal pressure gradient. Naturally, in regions were $\partial_{x} P$ is negligible, Eq. (2) reduces to the classical viscous sublayer equation, $u / u_{*}=z u_{*} / v$.

$$
U=(1 / 2 \mu) \partial_{x} P z^{2}+\left(\tau_{*} / \mu\right) z
$$

In summary, the wall-shear stress can then be estimated through three different methods: (i) through the slope of the velocity profile in the viscous sublayer (Eq. (2)), (ii) through the Clauser chart, assuming the existence of a logarithmic velocity distribution and (iii) through the average value of the total shear stress, considering the existence of a region adjacent to the wall where its distribution is nearly constant. The only method that can be used throughout the flow domain is method (i). In fact, we know that in regions of separated flow the classical logarithmic behaviour as well as the equilibrium conditions ceases to exist.

A particular concern to a correct application of Eq. (2) is the specification of an adequate coordinate system. For flows over a flat wall, the $x$-coordinate can be aligned with the mean flow direction, resulting in a rectangular Cartesian system where the momentum balance in $x$-direction contains most of the dynamical information regarding the flow. For flows over curved surfaces, however, identifying a coordinate system where the main coordinate axis is aligned with the flow direction is a problem. To overcome this difficulty, Finnigan (1983) suggests the use of physical streamlined coordinates. These coordinates are, however, difficult to use in separated flow regions. Thus, most of the data presented in literature for flows over hills follow a rectangular Cartesian system, just as we have presented our data in the previous section.

In our problem, the near-wall measurements that are to be considered for evaluation of the wall-shear stress in the flow separated region were made for distances smaller than $3 \mathrm{~mm}$ from the wall. In fact, in the first $3 \mathrm{~mm}$, eight points are to be considered for flow characterization. In our worst case scenario, the wall tangent corresponds to an angle of about $14^{\circ}$ (station $x / H=1.25$ ). Since $\sin \left(14^{\circ}\right)=0.24$, the corresponding streamwise velocity displacement along the normal direction will occur over a maximum distance of $0.7 \mathrm{~mm}$. In addition, the fact that $U \gg W$ close to the wall implies that the wall-shear stress can be calculated directly from the procedure stated above with the rectangular Cartesian 
system shown in Fig. 1. In Loureiro et al. (2007a), a detailed account of the measurement uncertainties is given.

\section{Turbulence models}

Six turbulence models will be investigated against the experimental data: four eddyviscosity models and two Reynolds stress models. The eddy-viscosity models are the standard $\kappa-\varepsilon$ model, the $\kappa-\omega$ model, the SST model and the RNG model. The two Reynolds stress models are the Speziale-Sarkar-Gatski (SSG) model and the baseline- $\omega$ model. The present selection of models is considered representative enough of the state of the art in turbulence engineering modelling to allow for a good assessment of the numerical computations of the flow over a hill.

The six chosen models are considered to be sufficiently well known to dispense a thorough description. Therefore, just a brief description of each method will be offered next. For further details the reader is referred to the original sources.

The equations of motion are the Reynolds averaged equations of continuity and momentum for an incompressible flow. Denoting the mean and fluctuating velocities in $x_{i}$ direction by $U_{i}$ and $u_{i}$, respectively, density and kinematic viscosity by $\rho$ and $v$, respectively, the equations can be written as

$$
\partial_{i} U_{i}=0, \quad \partial_{t} U_{i}+U_{j} \partial_{j} U_{i}=-\partial_{i} P+\partial_{j}\left[v\left(\partial_{j} U_{i}+\partial_{i} U_{j}\right)-\overline{u_{i} u_{j}}\right]
$$

where $P$ is the mean pressure divided by density.

\subsection{Eddy-viscosity models}

Eddy-viscosity models consider that the turbulent stresses, $\overline{u_{i} u_{j}}$, are related to the mean velocity gradients by a parameter of proportionality, the eddy or turbulent viscosity, $v_{t}$, according to

$$
-\overline{u_{i} u_{j}}=2 v_{t} S_{i j}-(2 / 3) \kappa \delta_{i j}, \quad S_{i j}=(1 / 2)\left(\partial_{j} U_{i}+\partial_{i} U_{j}\right)
$$

where $v_{t}$ denotes the eddy viscosity, $\kappa$ the turbulent kinetic energy and $\delta_{i j}$ is the Kronecker delta.

The eddy viscosity can be given different physical modelling. Many two-equation turbulence models consider the eddy viscosity to be related to the turbulent kinetic energy, $\kappa$, and to the dissipation of turbulent kinetic energy per unit mass, $\varepsilon$ through $v_{t}=C_{v} \kappa^{2} / \varepsilon$. Transport equations for $\kappa$ and for $\varepsilon$ can be derived directly from the Navier-Stokes equations through some algebraic manipulations and some extra subsequent modelling. The result is

$$
\begin{aligned}
& \partial_{i}\left(U_{i} \kappa\right)=\partial_{i}\left(\left(v+\left(v_{t} / \sigma_{\kappa}\right)\right) \partial_{i} \kappa\right)-\overline{u_{i} u_{j}} \partial_{j} U_{i}-\varepsilon \\
& \partial_{i}\left(U_{i} \varepsilon\right)=\partial_{i}\left(\left(v+\left(v_{t} / \sigma_{\varepsilon}\right)\right) \partial_{i} \varepsilon\right)-(\varepsilon / \kappa)\left(C_{\varepsilon 1} \overline{u_{i} u_{j}} \partial_{j} U_{i}+C_{\varepsilon 2} \varepsilon\right) .
\end{aligned}
$$

The model constants $C_{v}(=0.09), C_{\varepsilon 1}(=1.44), C_{\varepsilon 2}(=1.92), \sigma_{\kappa}(=1)$, and $\sigma_{\varepsilon}(=1.3)$ are given different values according to different authors. In fact, these constants are adjusted by different authors so as to warrant good model performance to specific applications. Corrections due to the Coriolis effects on turbulence, due to weak wind shear, or due to streamline curvature effects have also been incorporated in these constants by authors. 
A renormalization group analysis of the Navier-Stokes equation results in a set of transport equations - the RNG model-that is the same as that for the $\kappa-\varepsilon$ model. The model constants for the $\varepsilon$-equation, however, are different and are given, for example, by

$$
\begin{aligned}
& C_{\varepsilon 1 \mathrm{RNG}}=1.42-f_{\eta}, \quad f_{\eta}=\eta(1-\eta / 4.38) /\left(1+\beta_{\mathrm{RNG}} \eta^{3}\right), \\
& \eta=\sqrt{P_{i j} / \rho C_{\mu \mathrm{RNG}} \varepsilon},
\end{aligned}
$$

where $\beta_{\mathrm{RNG}}(=0.012)$ and $C_{\mu \mathrm{RNG}}(=0.085)$ must be adequately chosen and $P_{i j}$ denotes the production term.

Two equation models based on the $\kappa-\varepsilon$ formulation are known to suffer limitations in the description of the near-wall region since they tend to fail in predicting the correct functional near-wall behaviour of the logarithmic solution. A perturbation analysis of the $\kappa-\varepsilon$ formulation in the near-wall region shows that the standard constants yield solutions to the approximate equations that do not follow the classical law of the wall constants, $x=0.4$ and $A=5$. For this reason, a common practice is to use the $\kappa-\varepsilon$ model with boundary conditions specified not at the wall, but at some distance above the wall. In this case, the no-slip condition is then replaced by a wall function, the classical law of the wall.

An alternative two equation model that is claimed to circumvent this difficulty is the $\kappa-\omega$ model (Wilcox, 1988). In fact, the great advantage of the $\kappa-\omega$ formulation is supposed to be exactly the near-wall treatment, which can accept higher values of $z^{+}=\left(z u_{*} / v\right)$, the non-dimensional distance from the wall. The $\kappa-\omega$ model has the additional advantage of providing near-wall analytical solutions for both the viscous and the fully turbulent regions. In the $\kappa-\omega$ model, the eddy viscosity is taken as $v_{t}=(\kappa / \omega)$. The two transport equations for $\kappa$ and $\omega(=\varepsilon / \kappa)$ are then given by

$$
\begin{aligned}
& \partial_{i} U_{i} \kappa=\partial_{i}\left(\left(v+\sigma^{*} v_{t}\right) \partial_{i} \kappa\right)-\overline{u_{i} u_{j}} \partial_{j} U_{i}-\beta^{*} \kappa \omega, \\
& \partial_{i} U_{i} \omega=\partial_{i}\left(\left(v+\sigma v_{t}\right) \partial_{i} \omega\right)-\alpha(\omega / \kappa) \overline{u_{i} u_{j}} \partial_{j} U_{i}-\beta \omega^{2},
\end{aligned}
$$

where $\alpha\left(=\frac{5}{9}\right), \beta(=0.075), \beta^{*}(=0.09), \sigma(=0.5)$ and $\sigma^{*}(=0.5)$ are model constants.

Both models, the $\kappa-\varepsilon$ and the $\kappa-\omega$, fail to account for the transport of turbulent stress. The consequence is an over-prediction of the eddy viscosity that results in a poor prediction of the onset and the amount of flow separation from smooth surfaces. The SST$(\kappa / \omega)$ model proposes to account properly for the turbulent shear stress transport by considering

$$
v_{t}=\alpha \kappa /\left(\max \left(\alpha \omega, S_{i j} F_{S}\right)\right), \quad F_{S}=\tanh \left[\left(\max \left(\frac{2 \sqrt{\kappa}}{\beta^{*} \omega z}, \frac{500 v}{z^{2} \omega}\right)\right)^{2}\right],
$$

where $F_{S}$ is a function introduced to limit the turbulent viscosity.

\subsection{Second-moment models}

A more general way to model a turbulent flow is to compute every component of the Reynolds stress tensor from transport equations derived directly from algebraic manipulations of the Navier-Stokes equations. The resulting loss of information implied by the averaging process must then be recovered by an adequate modelling of each of the terms present in the equations. Most models consider the same basic set of rules to close 
the equations. All turbulent quantities are considered to be a function of Reynolds stress, $\kappa, \varepsilon$ (or alternatively $\omega$ ), mean flow quantities and related thermodynamics variables. The diffusion of turbulent quantities, in particular, is taken to be proportional to the local gradient of the quantity. The dissipation of turbulent kinetic energy is supposed to occur at very small scales where turbulence is isotropic.

Constants appearing in the models are ad hoc so that they must be fixed through experimental calibration. The models also need to be consistent with the common requirements of symmetry, invariance and permutation.

The resulting Reynolds stress model, formulated in terms of $\varepsilon$, can be written as

$$
\partial_{k} U_{k} \overline{u_{i} u_{j}}=\partial_{k}\left(\left(v_{t}+v\right) \partial_{k} \overline{u_{i} u_{j}}\right)+P_{i j}+\phi_{i j}-(2 / 3) \delta_{i j} \varepsilon
$$

where $P_{i j}$, the turbulence production term, and $\phi_{i j}$, the pressure-strain term, are given by

$$
P_{i j}=-\left(\overline{u_{i} u_{k}} \partial_{k} U_{j}+\overline{u_{j} u_{k}} \partial_{k} U_{i}\right)
$$

and

$$
\phi_{i j}=\phi_{1 i j}+\phi_{2 i j}
$$

where

$$
\begin{aligned}
\phi_{1 i j}= & -C_{s 1} \varepsilon a_{i j}-C_{s 2} \varepsilon\left(a_{i k} a_{j k}-(1 / 3) a_{m k} a_{k m} \delta_{i j}\right), \\
\phi_{2 i j}= & -C_{r 1} P_{i j} a_{i j}+C_{r 2} \kappa S_{i j}-C_{r 3} \kappa S_{i j}\left(a_{m k} a_{k m} \delta_{i j}\right)^{1 / 2} \\
& +C_{r 4} \kappa\left(a_{i j} S_{j i}+S_{i j} a_{j i}-(2 / 3) a_{k m} S_{m k} \delta_{i j}\right)+C_{r 5} \kappa\left(a_{i j} W_{j i}+W_{i j} a_{j i}\right)
\end{aligned}
$$

with

$$
a_{i j}=\left(\left(\overline{u_{i} u_{j}} / \kappa\right)-(2 / 3) \delta_{i j}\right), \quad W_{i j}=(1 / 2)\left(\partial_{j} U_{i}-\partial_{i} U_{j}\right)
$$

and where $C_{s 1}(=1.7), C_{s 2}(=-1.05), C_{r 1}(=0.9), C_{r 2}(=0.8), C_{r 3}(=0.65), C_{r 4}(=0.625)$ and $C_{r 5}(=0.2)$ are model constants.

The model can then be completely defined provided an equation for $\varepsilon$ (see, e.g., Eq. (6)) is used to close the system of partial differential equations. On the other hand, when the Reynolds stress model is based on the $\omega$ formulation, an equation for $\omega$ must be specified (see, e.g., Eq. (9)).

The large number of constants that appear in the Reynolds stress models allows for many different variations in formulation by authors. Launder et al. (1975) suggested models that use a pressure-strain linear correlation and isotropic and quasi-isotropic turbulence production. These models are normally termed LRR-IP and LRR-QI. An alternative model that uses a quadratic relation for the pressure-strain correlation was proposed by Speziale et al. (1991), the SSG model.

Despite the superior handling of the wall conditions, the $\kappa-\omega$ formulation struggles with its strong sensitivity to free stream conditions. Thus, given the different zonal strengths and, for that matter, weaknesses of the $\kappa-\varepsilon$ and the $\kappa-\omega$ formulations, a good balance can be achieved between both models if a blending is introduced between the $\kappa-\omega$ formulation near the surface and the $\kappa-\varepsilon$ model in the outer flow. This solution was proposed by Menter (1994), who introduced the so-called baseline $\kappa-\omega$ model (BSL). Therefore, the BSL-RSM- $\omega$ model proposes to multiply the Reynolds stress $\omega$-based model equation by a blending function $F_{\mathrm{B}}$ and the standard $\kappa-\varepsilon$ model equation by $1-F_{\mathrm{B}}$. Adding these two equations together, the resulting equation will have coefficients with the form 
$\phi_{\mathrm{BSL}}=F_{\mathrm{B}} \phi_{\omega}+\left(1-F_{\mathrm{B}}\right) \phi_{\varepsilon}$, thus reducing to the limiting RSM- $\omega$ and $\kappa-\varepsilon$ forms near and far away from the wall, respectively. Typically, $F_{\mathrm{B}}=\tanh \left(\mathrm{arg}^{4}\right)$ with

$$
\arg =\min \left[\max \left(\frac{\sqrt{\kappa}}{\beta^{*} \omega z}, \frac{500 v}{z^{2} \omega}\right), \frac{4 \sigma^{\prime} \rho \kappa}{C D_{\kappa \omega} z^{2}}\right]
$$

and

$$
C D_{\kappa \omega}=\max \left(2 \rho\left(\sigma^{\prime} / \omega\right) \nabla \kappa \nabla \omega, 10^{-10}\right),
$$

where $\sigma^{\prime}=1 / 0.856$.

\section{Computational details}

The equations governing the problem were solved using the well-known code ANSYS CFX, release 5.7. The model solves the Reynolds averaged Navier-Stokes equations (RANS) through a finite-volume approach. The solution strategy consists in solving the momentum equations using a guessed pressure. Next, a pressure correction is obtained which typically needs a large number of iterations to reach a converged solution. The code uses a coupled solver that solves the equations for the flow parameters as a single system. This procedure uses a fully implicit discretization of the equations at any given time. In the present steady state case, the time step behaves like an acceleration parameter to find the approximate solutions in a physically meaningful framework to a time-independent solution.

The use of wall functions has been almost a standard procedure in numerical codes. Since viscous effects are always confined to a very thin wall adjacent region, a common approach to escape from resolving the large gradients in flow variables is to appeal to analytical solutions that are valid in the fully turbulent region. These analytical solutions, usually termed wall functions, can then be used to provide adequate near-wall conditions without having to resolve the viscous region. In the classical approach (Launder and Spalding, 1974), the near-wall longitudinal velocity is related to the wall-shear stress through a log-relation.

To avoid the log-relation to become singular at separation points where the wall-shear stress is zero, a specific strategy has to be devised. An alternative approach is to adopt $u_{s}=C_{v}^{1 / 4} \kappa^{1 / 2}$ as the velocity scale in the logarithmic term. Then, $u_{*}$ can be computed directly from the log-relation. The problem with wall functions is that predictions are strongly dependent on mesh features; in particular, mesh refinement does not provide solutions with increasing accuracy.

For the $\kappa-\varepsilon$ model of the ANSYS CFX code, this problem is overcome by the so-called scalable wall functions. In this approach, the $z^{+}$value used in the log-relation is limited to $\max \left(z^{+}, 11.06\right)$. Therefore, mesh inconsistencies are avoided by considering in the calculations only the mesh points that are located outside the viscous sublayer. Scalable wall functions have the advantage of being applicable to arbitrarily fine meshes.

An alternative procedure to flow simulation is to consider models that incorporate a wall damping effect. These models are termed low-Reynolds number models. Typically, a low$R \kappa-\varepsilon$ model requires a mesh resolution of $z^{+}<0.2$. For this reason, their use is very consuming in terms of computer-storage and runtime. As we shall see, in the present work the typical near-wall mesh size lies in the range $1.1<z^{+}<1.7$. Therefore, a low-Reynolds number solution strategy for the $\kappa-\varepsilon$ model will not be used here. 
As for the $\omega$-based models, an automatic near-wall treatment can be used. The $\kappa-\omega$ formulation of Wilcox (1988) has the advantage of having analytical solutions in the logarithmic and in the viscous regions. In the automatic near-wall treatment, these two solutions are blended smoothly. The implication is that the first mesh point can then be dislocated to the viscous sublayer. Wall functions are replaced by a low Reynolds number formulation once a near-wall grid resolution of at least $z^{+}<2$ is achieved. This fact, allied to the $\omega$ analytical solution in the viscous sublayer, constitutes a significant advantage in comparison to the $\kappa-\varepsilon$ model. The automatic switch to a low- $R$ model is best resolved when at least 10 nodes are located in the near-wall turbulent and viscous regions.

The coordinate system used for the computations was the same defined for the experiments, as shown in Fig. 1. The upstream and downstream boundaries extended from $x / H=-12.5$ to 10 . The upper boundary was defined at $z / H=3.93$. A two-dimensional computation was achieved by defining just two lateral boundaries at $y= \pm 0.5 \mathrm{~mm}$.

An extensive grid-dependence test was performed resulting in a final non-uniform, bodyfitted mesh with 110,376 elements. The grid evaluation test consisted of successive computations that used systematically refined meshes. The computation whose results did not vary between two consecutive refinements indicated the most suitable mesh. A grid with $300 \times 1 \times 80$ cells was found to suffice to resolve the flow in the bottom subregions II and III, as illustrated in Fig. 2. The mesh was particularly refined in the near-wall region so as to completely resolve the inner turbulent and viscous sublayers. In fact, the computational domain consisted of a two-block, structured mesh. The first block is $z / H=$ 1.33 in height and covers the bottom region of the domain. The second block covers the remaining $z / H=2.6$ upper region of the domain.

To obtain further flexibility in defining the grid characteristics, each block was divided into four subregions, two upstream and two downstream of the top of the hill. In $z$-direction, the nearest node, $z_{p}$, was $0.25 \mathrm{~mm}$ away from the wall. Under this condition, the value of $z_{p}^{+}\left(=z_{p} u_{*} / v\right)$, varied between 1.1 and 1.7 , from stations $x=0$ to $300 \mathrm{~mm}$. The cell-aspect ratios in these regions are 1.0/1.0/1.0. In the upper region of the computational domain, a relatively coarse mesh was used, with a maximum cell-aspect ratio of 8.0/1.0/4.0. In these regions, the grid expansion ratio does not exceed 1.01 and 1.03 in $x$ - and $z$-directions, respectively.

Inlet conditions were prescribed directly from the experimental data, including the mean and the fluctuating quantities. At the outlet, the turbulent intensity was automatically

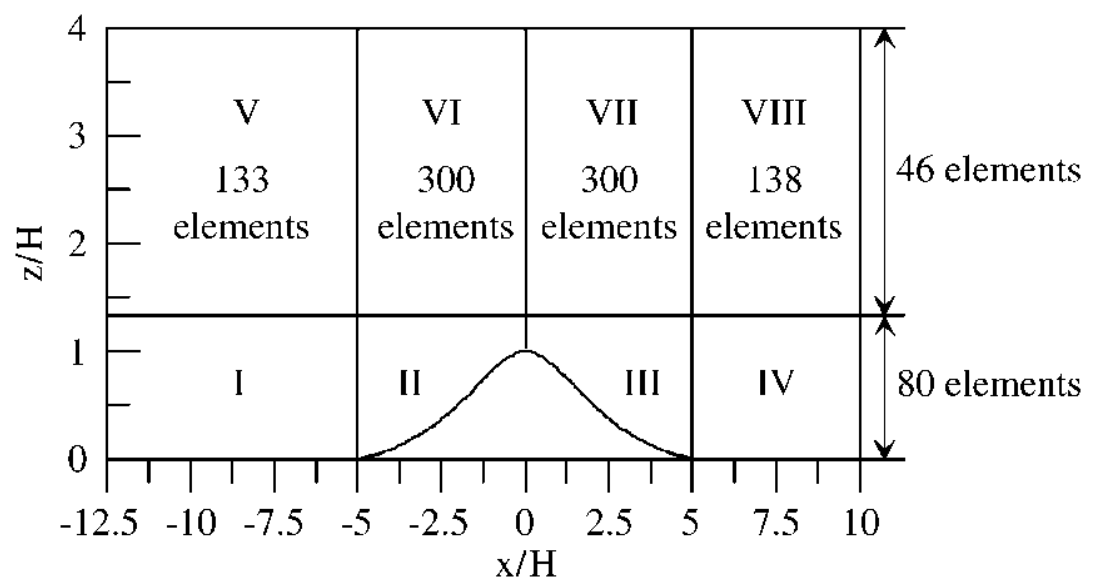

Fig. 2. Computational domain. 
Table 3

Computational times

\begin{tabular}{|c|c|c|c|c|c|c|}
\hline Model & $\kappa-\varepsilon$ & $\kappa-\omega$ & $R N G$ & $S S T$ & $S S G$ & $B S L$ \\
\hline Time & $27 \mathrm{~min}$ & $1 \mathrm{~h} 01 \mathrm{~min}$ & $31 \mathrm{~min}$ & $1 \mathrm{~h} 06 \mathrm{~min}$ & $51 \mathrm{~min}$ & $4 \mathrm{~h} 37 \mathrm{~min}^{*}$ \\
\hline
\end{tabular}

*Four machines in parallel processing.

calculated by the model. At the side walls a symmetry condition was imposed. At ground level, the no-slip boundary condition was used.

The computations were performed on a Pentium 4, 3.0 GHz, with $1 \mathrm{~Gb}$ DDR400 RAM operating in dual channel mode. Typical computational times are given in Table 3.

\section{Results}

As mentioned before, six different turbulence models were tested for the present geometry. Results will be reported for four eddy-viscosity models, standard $\kappa-\varepsilon, R N G, \kappa-\omega$ and $S S T$ models, and then, for two shear stress transport models, $S S G$ and $B S L$ Reynolds stress models.

The experimental and the computed general flow patterns are shown in Fig. 3. The most obvious and apparent conclusion is that none of the $\varepsilon$-based models were capable of predicting flow separation. This observation is quite different from those of Kobayashi et al. (1994), of Castro and Apsley (1997), of Kim et al. (1997) and of Kim and Patel (2000) who used $\varepsilon$-based models that did predict a reverse flow region. The simulations of Kobayashi et al. (1994) used a modified $\kappa-\varepsilon$ model proposed by Svensson and Haggkvist (1990) that included additional drag force terms. Castro and Apsley (1997) report that all three models they used, the standard $\kappa-\varepsilon$, a model with curvature corrections and a model with dissipation modifications, furnished separation and reversed flow. Kim et al. (1997) used the standard and the RNG-based $\kappa-\varepsilon$ models with a low-Reynolds number model. Kim and Patel (2000) were capable of predicting the separated flow region thanks to the specification of a low-Reynolds number model that resolved the flow in the viscous sublayer. Loureiro et al. (2007b) showed how three different near-wall treatments for the $\kappa-\varepsilon$ model resulted in very good predictions for the reverse flow region on the lee of a hill. As explained before, since our first grid point $\left(z_{p}^{+}=z_{p} u_{*} / v\right)$ lies between 1.1 and 1.7, ANSYS CFX uses just a scalable wall function, and not a low-Reynolds number model, to describe the inner layer of the flow. This solution strategy, thus, was not capable of predicting flow separation.

Although not entirely clear from Fig. 3a, the flow reattachment point was found experimentally to be located at about $x / H=6.67$. This point was estimated by measuring the $x$-velocity component over a distance $z / H=0.016$ away from the wall. When the velocity was observed to change from negative to positive over long sampling times, the length of the separated flow region was estimated. The best prediction for the flow separated region was given by the $\kappa-\omega$ model, followed closely by $S S T$ model. In fact, all three $\omega$-based models performed reasonably well. However, the BSL- $\omega$-RSM furnished the less agreeable prediction. The results for separated flow prediction are summarized in Table 4.

Selected mean velocity profiles at stations $x / H=0,3.75$ and 10 are shown in Fig. 4 . Very clearly, the strong flow acceleration on the top of the hill $(x / H=0)$ is not well 


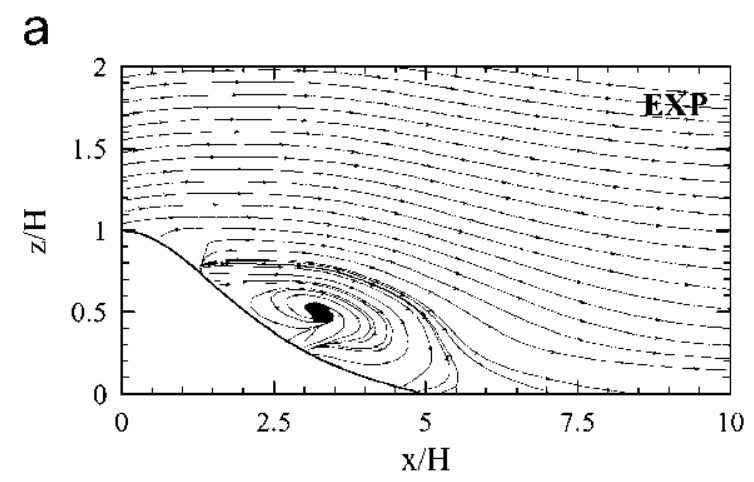

b

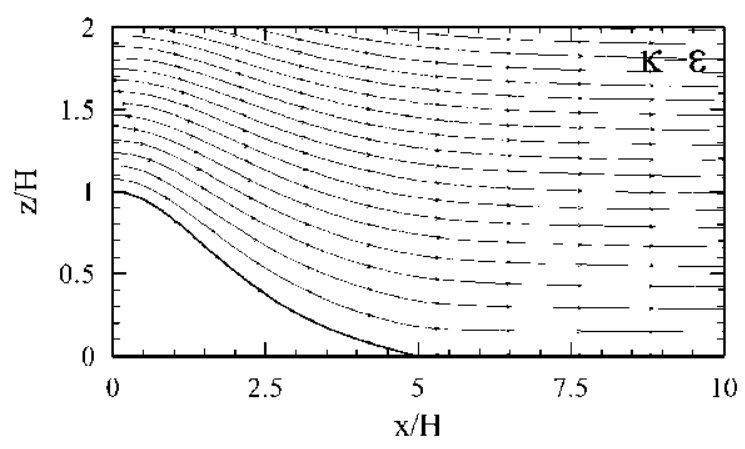

d

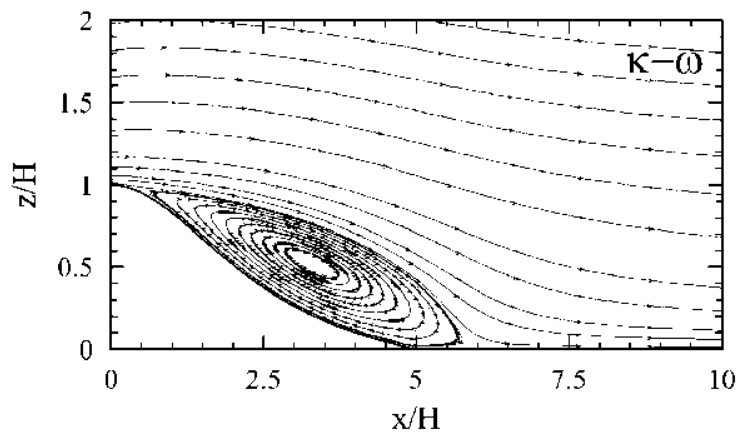

$f$

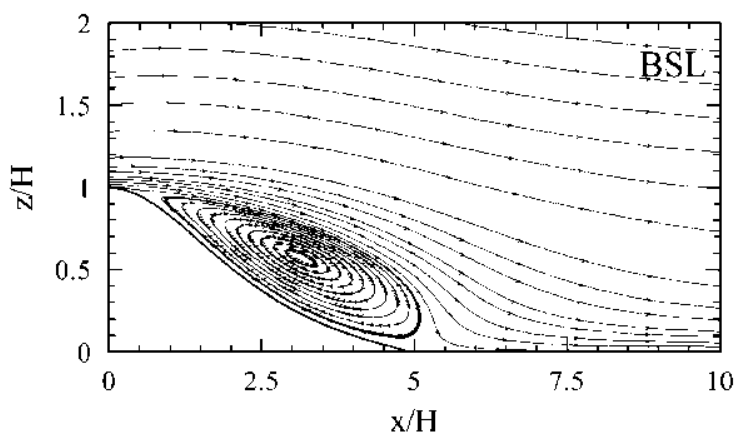

C

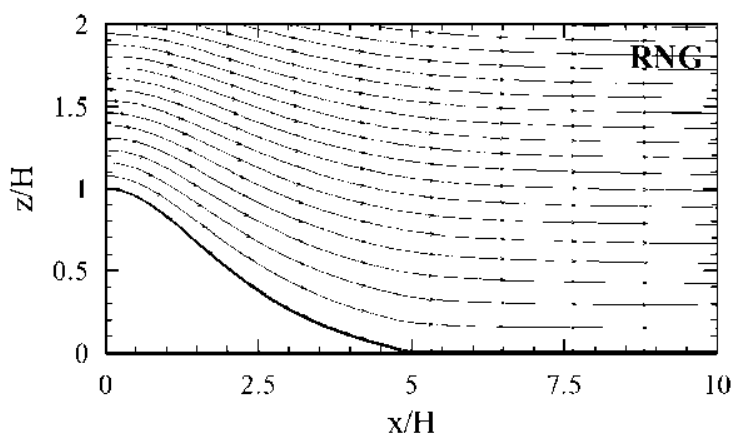

e

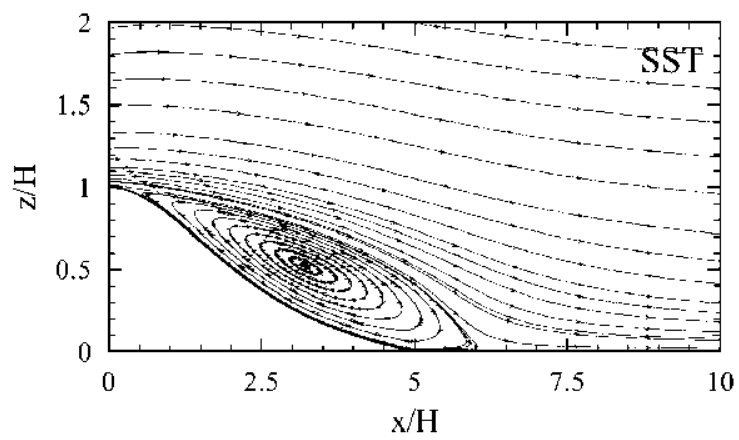

g

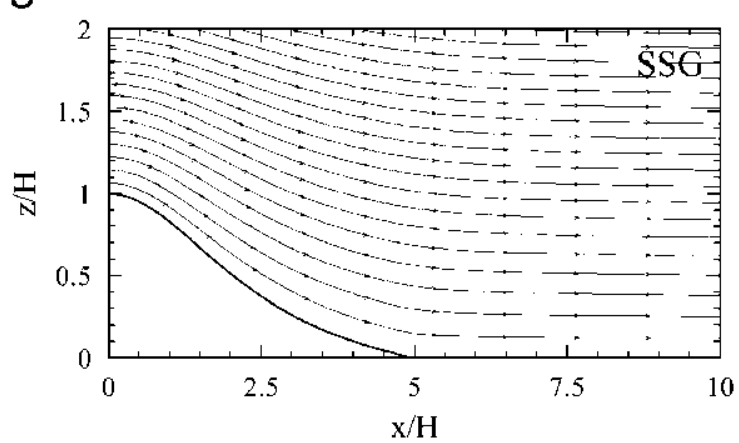

Fig. 3. Flow streamlines.

captured by any of the present models. Models tend to overestimate the velocity in the near-wall region. The result is a poor prediction of the speed-up factor, with a severe overestimation of its numerical value. Similar observations were made by Kobayashi et al. (1994), by Castro and Apsley (1997) and by Ross et al. (2004). The excessive velocity gradient in the wall region also results in a largely overestimated wall-shear stress. 
Table 4

Length of separated flow $(L / H)$ according to predictions

\begin{tabular}{llll}
\hline Model & Separation point $(x / H)$ & Reattachment point $(x / H)$ & $L / H$ \\
\hline$\kappa-\omega$ & 0.5 & 5.6 & 5.1 \\
$S S T$ & 0.53 & 5.53 & 5.0 \\
$B S L$ & 0.47 & 5.33 & 4.86 \\
Experiments & 0.5 & 6.67 & 6.17 \\
\hline
\end{tabular}
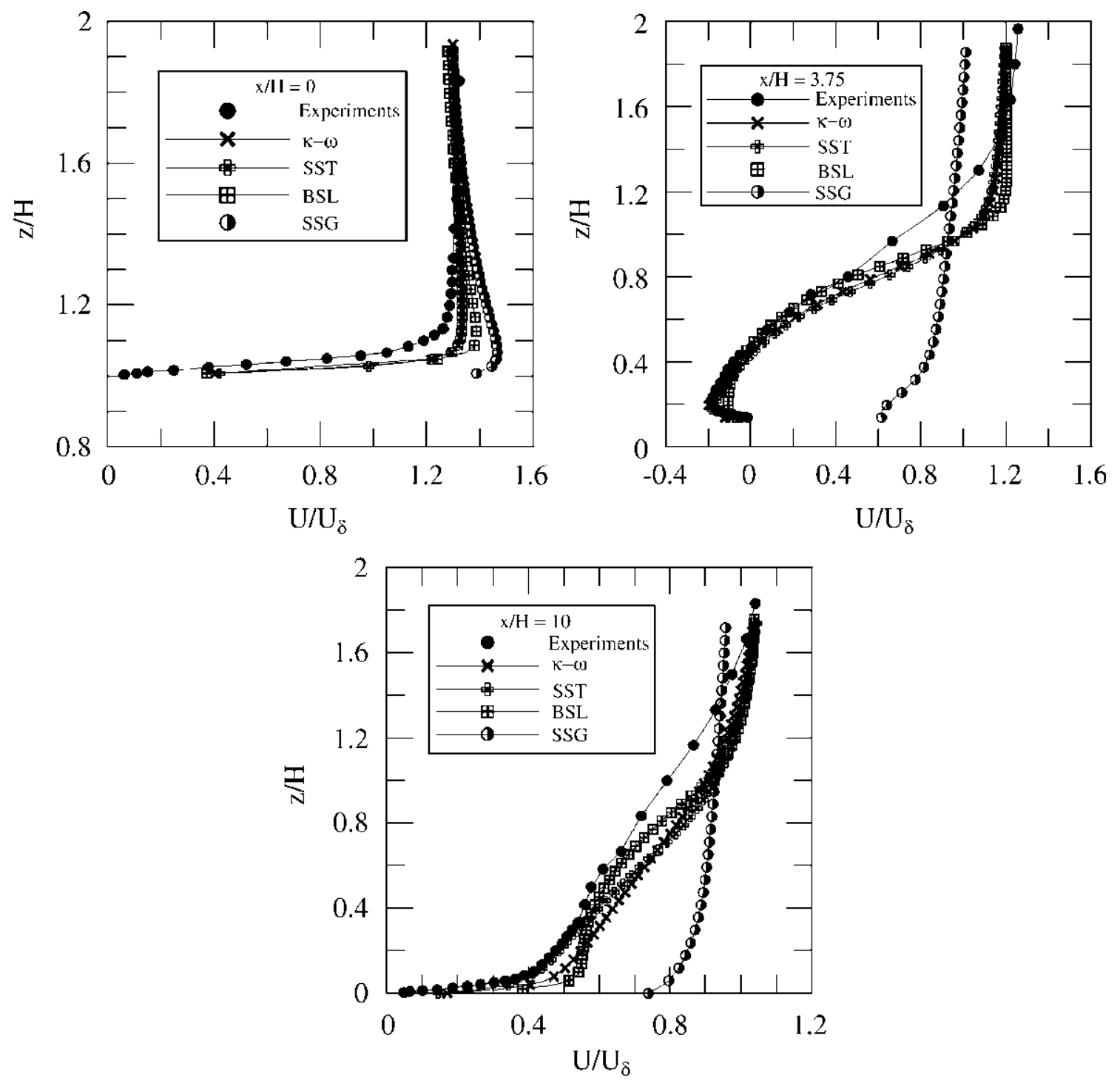

Fig. 4. Mean velocity profiles for stations $x / H=0,3.75$ and 10 .

In the reversed flow region $(x / H=3.75)$, very close to the wall $(z / H<0.35)$, the $\kappa-\omega$ and the $S S T$ models perform better than the others. However, in the region of positive flow velocity $0.35<z / H<0.9$, the $B S L$ model offers the best performance. At station $x / H=10$, the best agreement provided by the $S S T$ model in the near-wall region $(z / H<0.4)$ is apparent. The $B S L$ model is best over the short interval $0.4<z / H<0.7$. 
The components of the Reynolds stress tensor are shown in Figs. 5-7 for the same selected stations, $x / H=0,3.75$ and 10 . As anticipated by the mean velocity results, all models present major difficulties in trying to predict the turbulent flow properties on the hill top. The general pattern of the turbulence characteristics of the flow are not captured anywhere by the simulations. This result is difficult to interpret from the contour graphs of Ross et al. (2004) due to their low resolution. Iizuka and Kondo (2004) do show graphs of the turbulent kinetic energy, but the individual behaviour of the Reynolds stress tensor components is not shown.

In fact, at the hill summit, the longitudinal velocity profile is nearly uniform with very little shear. All turbulence model simulations, however, furnish a monotonic increase of $\overline{u u}$ with wall distance. In the flow separated region, turbulence profiles are characterized by an elevated maximum whose distance to the wall increases with increasing distance from the hill. In our experiment, an increase of $\overline{u u}$ of the order of four times is observed. This increase is well predicted by the $B S L$ model. In particular, the peak value for $\overline{u u}$, located at $x / H=3.75, z / H=0.8$, near the centre of the recirculation bubble is well reproduced. The $\kappa-\omega$ and the SST models do manage to find the right location where $\overline{u u}$ peaks to a
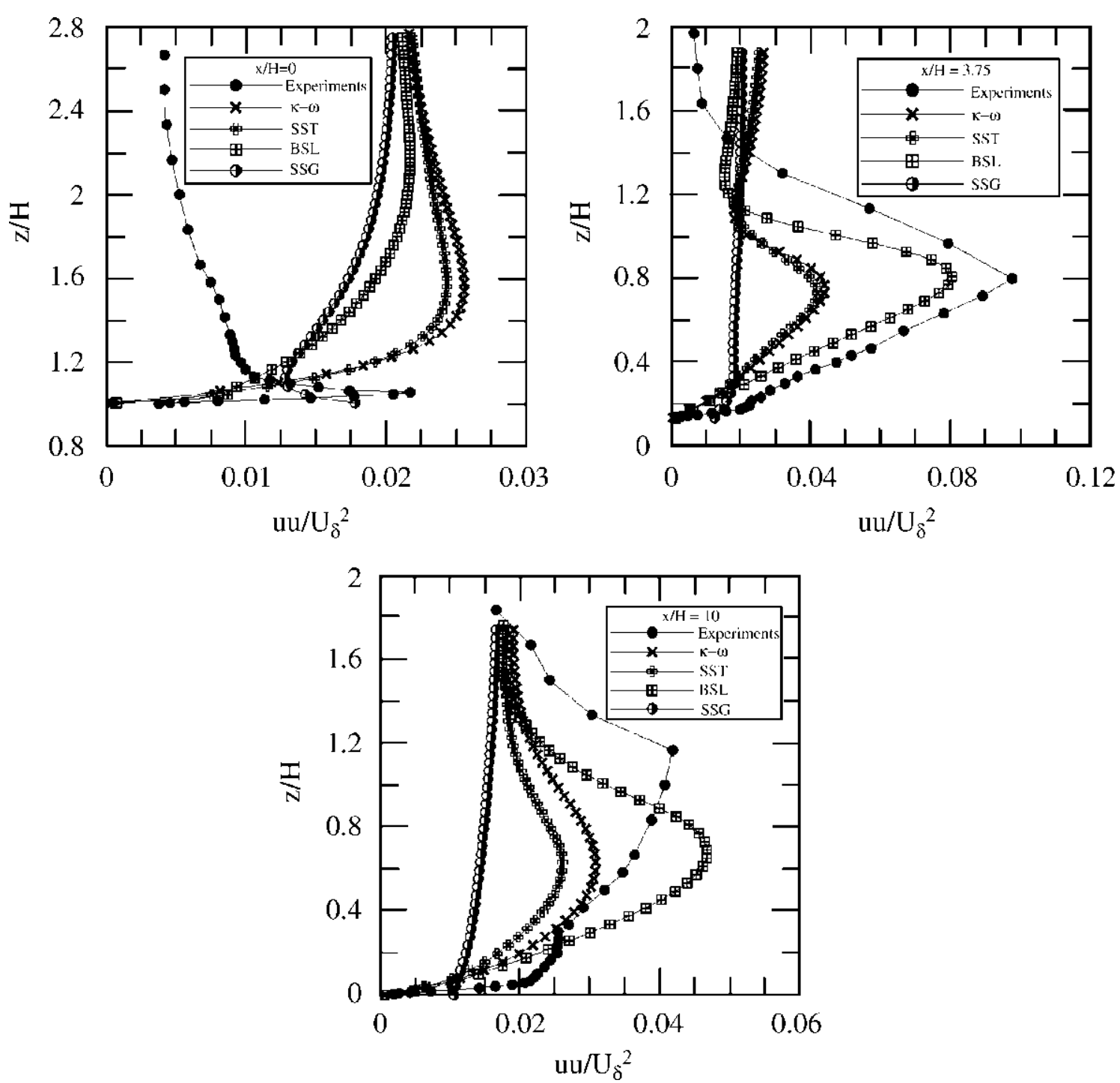

Fig. 5. Turbulence profiles, $\overline{u u}$, for stations $x / H=0,3.75$ and 10 , respectively. 

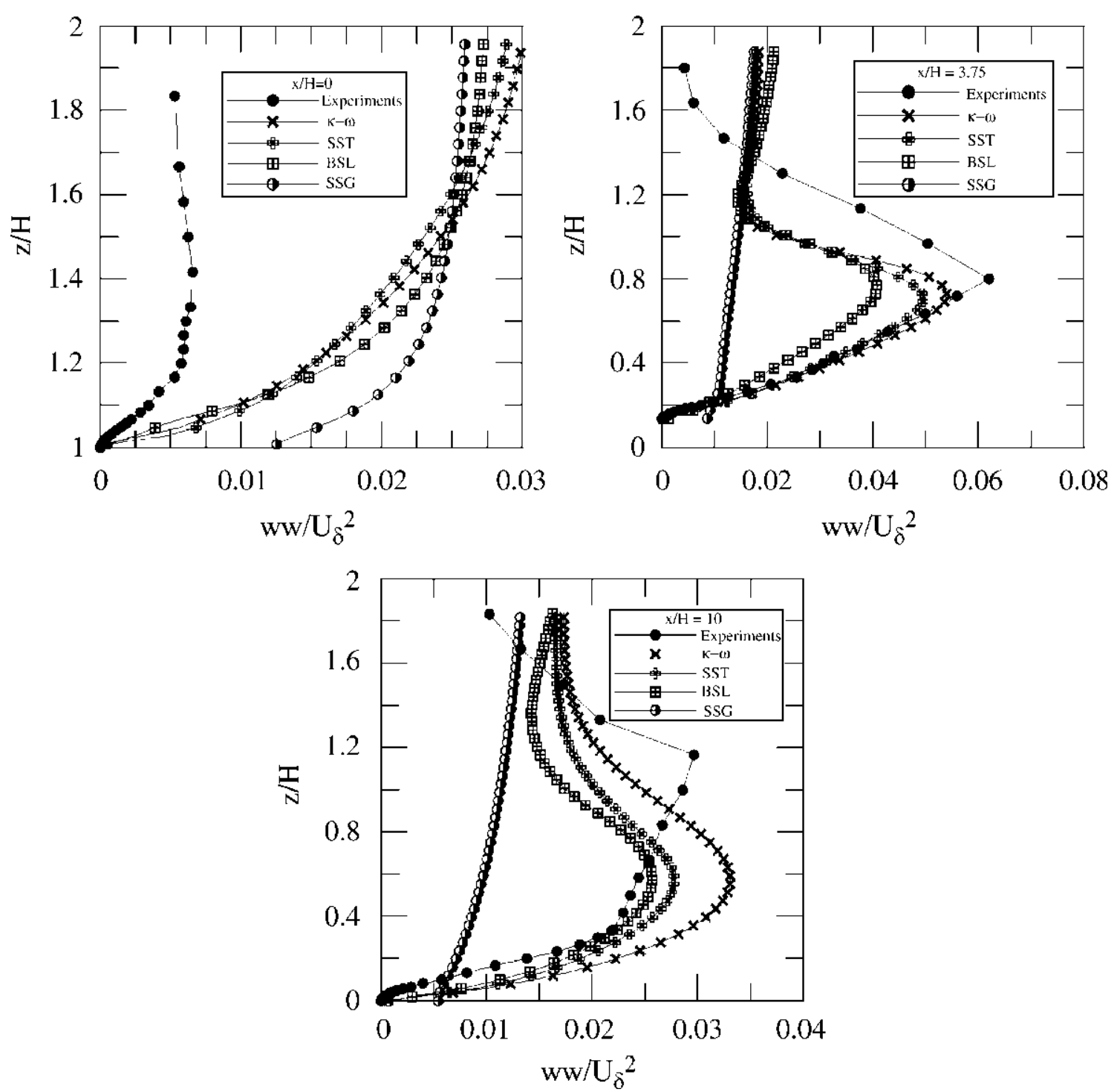

Fig. 6. Turbulence profiles, $\overline{w w}$, for stations $x / H=0,3.75$ and 10 , respectively.

maximum, but predicted values that are less than half of the measured values. Downstream of the hill, at station $x / H=10$, the $B S L$ model still provides the best results. However, the location of maximum $\overline{u u}$ is largely in error.

On the hill top, $\overline{w w}$ is observed to experience a relative increase of about $50 \%$ as compared to the undisturbed values. Since a large increase in $W$ is observed uphill, the larger values of $\overline{w w}$ may be explained by the increase in turbulence production through the term $\Pi_{w w}=-2 \overline{u w}(\partial W / \partial x)$. This increase is observed to be followed by a further, and much stronger increase, of about 20 fold in the separated flow region. The distribution of $\overline{w w}$ follows the same pattern distribution of $\overline{u u}$ across the reversed flow region, although $\overline{w w}$ is found to be about $65 \%$ of $\overline{u u}$. In special, the maximum value of $\overline{w w}$ is also located at $x / H=3.75, z / H=0.8$. Much in the same way as for the $\overline{u u}$ results, the numerical predictions of $\overline{w w}$ provided by all models at the hill summit are very poor. Again, not even the general trends of the $\overline{w w}$-profiles are correctly given. At station $x / H=3.75$, the $\kappa-\omega$ and the SST models provide the best results, the measured profile is reasonably well reproduced and the maximum value of $\overline{w w}$ and its location are reasonably well captured. 

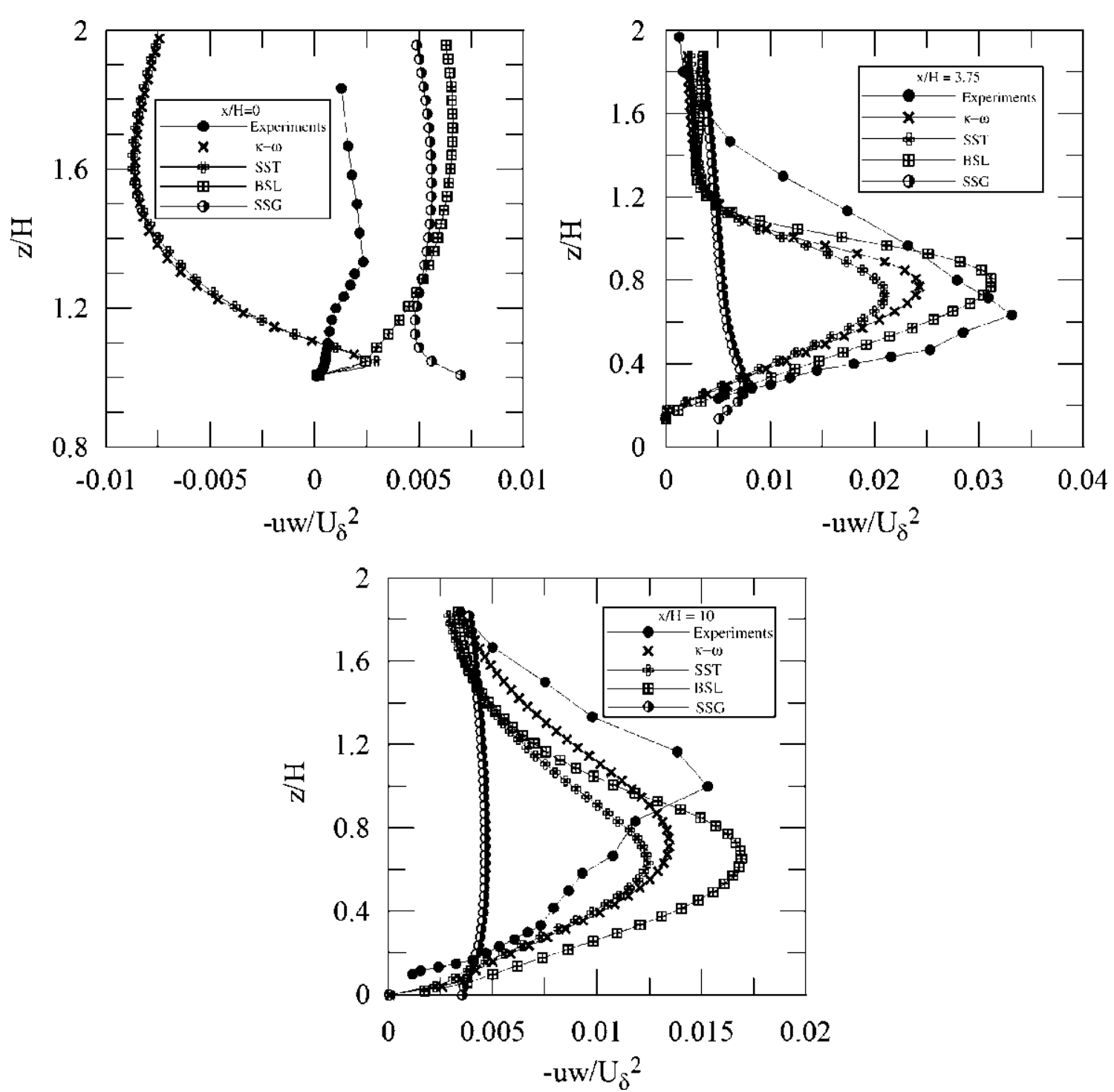

Fig. 7. Turbulence profiles, $-\overline{u w}$, for stations $x / H=0,3.75$ and 10 , respectively.

At station $x / H=10$, the $\kappa-\omega$ and the $S S T$ models give the best predictions for the maximum value of $\overline{w w}$. However, its peak location is not rightly guessed.

The changes in Reynolds shear stress profile on the top of the hill are relatively small, varying very little with height. This behaviour has not been identified by any of the turbulence models. The $\kappa-\omega$ and the $S S T$ models even give unrealistic predictions, exhibiting negative values of $-\overline{u w}$. At station $x / H=3.75$, a large increase of the order of 17 times in $-\overline{u w}$ is observed. This behaviour may be explained by the enhanced shear effects through the production term $\Pi_{u w}=\overline{w w}(\partial U / \partial z)$. The predictions given by the $B S L$ model reproduce well the $-\overline{u w}$ profile, guessing to a good degree of certainty the value and the position of maximum $-\overline{u w}$. The $\kappa-\omega$ and the $S S T$ models underestimate $-\overline{u w}$ by about $30 \%$. At station $x / H=10$, the $B S L$ model provides the best results but, on the other hand, overestimates $\overline{u w}$. The computed location of the maximum of $-\overline{u w}$ is well below the measured value.

The wall-shear stress distribution is shown in Fig. 8. Experimental data obtained through the procedure explained in Section 2.4 are denoted by $(+)$; the curve that connects 


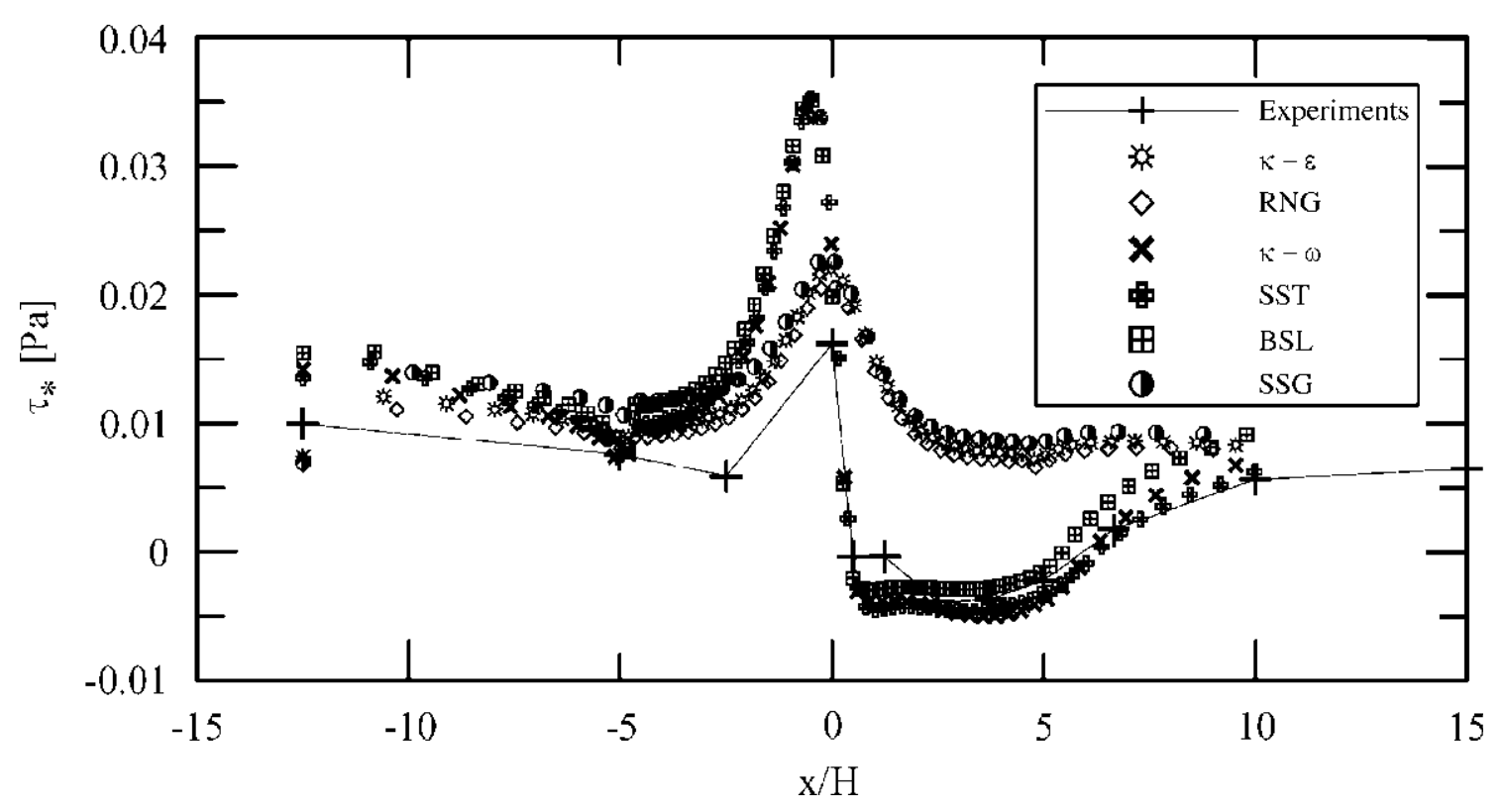

Fig. 8. Wall-shear stress predictions.

these symbols has been added to improve legibility. Data of all six different numerical simulations are also shown in Fig. 8. Upstream of the hill all simulations performed well. On the hill top, the inability of the models to resolve the near-wall layers resulted in a large overestimation of $\tau_{*}$. This is a direct consequence of the largely overestimated near-wall velocity gradients. In the region of reverse flow, models $\kappa-\varepsilon, R N G$ and $S S G$ did not furnish any consistent result. In special, as we have mentioned before, they were not even capable of predicting flow separation. On the other hand, models $\kappa-\omega, S S T$ and $B S L$ gave very good results. The high peak in $\tau_{*}$ was not particularly captured by the experiments. Hence, we could not perform any more detailed comparison at this point. However, the general levels of $\tau_{*}$ in the regions of attached as well as reverse flow are very well predicted. The value of minimum $\tau_{*}$ is almost exactly predicted by models $\kappa-\omega, S S T$ and $B S L$.

\section{Final remarks}

The present work has found that predictions of the flow over a steep hill for both the mean velocity and turbulent fields obtained with $\varepsilon$-based models were in poor agreement with experimental results mainly in what concerns the solution description in the reverse flow region. In particular, the $\varepsilon$-based models completely failed to predict the recirculation region. This is not a new information, for previous authors have shown that if $\varepsilon$-based models are expected to furnish good predictions for flows with separated regions, the standard treatment of the near-wall conditions has to be specialized. The simulation strategy adopted by the code ANSYS CFX for $\varepsilon$-based models, i.e., the scalable wall function, did not perform well with the present flow geometry. On the other hand, we have seen that results provided by the $\omega$-based models, which automatically switch from a wall function to a low-Reynolds number formulation, were in reasonable agreement with experiments.

Regarding just the mean properties of the flow, velocity and wall-shear stress, the SST model gave the best results. As far as the turbulence properties are concerned, the $B S L-R S M$ model gave the best results. All closure model based on the $\omega$-equation were 
reasonably successful in predicting the separation point and the properties of the flow inside the recirculation region. The peak values of $\overline{u u}, \overline{w w}$ and $-\overline{u w}$ predicted by the $B S L-R S M$ model were accurate to within $20 \%$. Still, considering the flow recirculation region, the $\kappa-\omega$ and $S S T$ models under-predicted turbulence properties by about $30-50 \%$.

The hill top was the specific region of the flow where computations really struggled to provide good predictions. Indeed, none of the flow properties were well predicted there. The sharp mean velocity gradients on the hill top were not captured by any of the models despite the very fine computational mesh that was deployed here. Turbulence results were completely out of the general experimental trends and were wrong by an order of magnitude.

To specify the boundary conditions, including the turbulence data, profiles were taken directly from the experiments. The reasonable prediction of turbulence data for the reverse flow region reflects, in some way, the adequate choice of boundary conditions.

\section{Acknowledgements}

A.P.S.F. is grateful to the Brazilian National Research Council (CNPq) for the award of a research fellowship (Grant no. 304919/2003-9). The work was financially supported by CNPq through Grant no. 472215/2003-5 and by the Rio de Janeiro Research Foundation (FAPERJ) through Grants E-26/171.198/2003 and E-26/152.368/2002. J.B.R.L. benefited from a Research Scholarship from the Brazilian Ministry of Education through CAPES. J.B.R.L. is also grateful to Programme Alban, European Union Programme of High Level Scholarships for Latin America, No. E03M23761BR, for further financial support. Authors are thankful to Prof. Fernando T. Pinho and to Prof. Maria Fernanda Proença for their support in making the experimental campaign possible, and to the staff of the Laboratory of Hydraulics of Oporto University for all their help in setting up the experimental facilities as well as for some useful discussions.

\section{References}

Arya, S.P.S., Capuano, M.E., Fagen, L.C., 1987. Some fluid modelling studies of flow and dispersion over twodimensional low hills. Atmos. Environ. 21, 753-764.

Britter, R.E., Hunt, J.C.R., Richards, K.J., 1981. Air flow over a two-dimensional hill: studies of velocity speedup, roughness effects and turbulence. Q. J. R. Meteorol. Soc. 107, 91-110.

Castro, F.A., Palma, J.M.L.M., Silva Lopes, A., 2002. Simulation of the Askervein flow. Part 1: Reynolds averaged Navier-Stokes equations k-e turbulence model. Boundary-Layer Meteorol. 107, 501-530.

Castro, I.P., Apsley, D.D., 1997. Flow and dispersion over topography: a comparison between numerical and laboratory data for two-dimensional flows. Atmos. Environ. 31, 839-850.

Finnigan, J.J., 1983. A streamlined coordinate system for distorted turbulent shear flows. J. Fluid Mech. 130, $241-258$.

Hewer, F.E., 1998. Non-linear numerical model predictions of flow over an isolated hill of moderate slope. Boundary-Layer Meteorol. 87, 381-408.

Hurley, P.J., 1997. An evaluation of several turbulence schemes for the prediction of mean and turbulent fields in complex terrain. Boundary-Layer Meteorol. 83, 43-73.

Iizuka, S., Kondo, H., 2004. Performance of various sub-grid scale models in large-eddy simulations of turbulent flow over complex terrain. Atmos. Environ. 38, 7083-7091.

Ishihara, T., Fujino, Y., Hibi, K., 2001. A wind tunnel study of separated flow over a two-dimensional ridge and a circular hill. J. Wind Eng. (Proceedings of the Fifth Asia-Pacific Conference on Wind Engineering, Kioto, Japan) 89, 573-576. 
Khurshudyan, L.H., Snyder, W.H., Nekrasov, I.V., 1981. Flow and dispersion of pollutants over two dimensional hills, Environmental Protection Agency Report No. EPA-600/4-81-067, Research Triangle Park, NC, p. 130.

Kim, H.G., Patel, V.C., 2000. Test of turbulence models for wind flow over terrain with separation and recirculation. Boundary-Layer Meteorol. 94, 5-21.

Kim, H.G., Lee, C.M., Lim, H.C., Kyong, N.H., 1997. An experimental and numerical study on the flow over two-dimensional hills. J. Wind Eng. Ind. Aerodyn. 66, 17-33.

Kobayashi, M.H., Pereira, J.C.F., Siqueira, M.B.B., 1994. Numerical study of the turbulent flow over and in a model forest on a 2D hill. J. Wind Eng. Ind. Aerodyn. 53, 357-374.

Launder, B.E., Spalding, D.B., 1974. The numerical computation of turbulent flows. Comput. Methods Appl. Mech. Eng. 3, 269-289.

Launder, B.E., Reece, G.J., Rodi, W., 1975. Progress in the developments of a Reynolds stress turbulence closure. J. Fluid Mech. 68, 537-566.

Logan, S.E., 1972. A laser velocimeter for Reynolds stress and other turbulence measurements. AIAA J. 10, 933-935.

Loureiro, J.B.R., Pinho, F.T., Silva Freire, A.P., 2007a. Near wall characterization of the flow over a twodimensional steep smooth hill. Exp. Fluids 42, 441-457.

Loureiro, J.B.R., Soares, D.V., Fontoura Rodrigues, J.L.A., Pinho, F.T., Silva Freire, A.P., 2007b. Water tank and numerical model studies of flow over steep smooth two-dimensional hills. Boundary-Layer Meteorol. 122, 343-365.

Menter, F.R., 1994. Two-equation eddy-viscosity turbulence models for engineering applications. AIAA J. 32, $1598-1605$.

Ross, A.N., Arnold, S., Vosper, S.B., Mobbs, S.D., Nixon, N., Robins, A.G., 2004. A comparison of wind-tunnel experiments and numerical simulations of neutral and stratified flow over a hill. Boundary-Layer Meteorol. $113,427-459$.

Ruck, B., Adams, E., 1991. Fluid mechanical aspects of the pollutant transport to coniferous trees. BoundaryLayer Meteorol. 56, 163-195.

Speziale, C.G., Sakar, S., Gatski, T.B., 1991. Modelling the pressure-strain correlation of turbulence: an invariant dynamical systems approach. J. Fluid Mech. 227, 245-272.

Svensson, V., Haggkvist, K., 1990. A two equation turbulent model for canopy flows. J. Wind Eng. Ind. Aerodyn. $35,201-211$.

Wang, C., Jang, Y.J., Leschziner, M.A., 2004. Modelling two- and three-dimensional separation from curved surfaces with anisotropy-resolving turbulence closures. Int. J. Heat Fluid Flow 25, 499-512.

Wilcox, D.C., 1988. Multiscale model for turbulent flows. AIAA J. 26, 1311-1320.

Ying, R., Canuto, V.M., 1997. Numerical simulation of the flow over two-dimensional hills using a second-order turbulence closure model. Boundary-Layer Meteorol. 85, 447-474. 